\title{
Some companions of perturbed Ostrowski-type inequalities based on the quadratic kernel function with three sections and applications
}

Wenjun Liu' ${ }^{1 *}$, Yiting Zhu ${ }^{1}$ and Jaekeun Park ${ }^{2}$

"Correspondence:

wjliu@nuist.edu.cn

${ }^{1}$ College of Mathematics and

Statistics, Nanjing University of

Information Science and

Technology, Nanjing, 210044, China

Full list of author information is

available at the end of the article

\begin{abstract}
In this paper, based on the quadratic kernel function with three sections, which was defined by Liu in 2009, we establish some companions of perturbed Ostrowski-type inequalities for the case when $f^{\prime \prime} \in L^{1}[a, b], f^{\prime \prime \prime} \in L^{2}[a, b]$ and $f^{\prime \prime} \in L^{2}[a, b]$, respectively. The special cases of these results offer better estimation than the conventional trapezoidal formula and the midpoint formula. The results we get can apply to composite quadrature rules in numerical integration and probability density functions. The effectiveness of these applications is also illustrated through several specific examples due to better error estimates.
\end{abstract}

MSC: 26D15; 41A55; 41A80; 65C50

Keywords: perturbed Ostrowski type inequality; differentiable mapping; composite quadrature rule; probability density function

\section{Introduction}

In 1938, Ostrowski [1] established the following interesting integral inequality for differentiable mappings with bounded derivatives.

Theorem 1.1 Let $f:[a, b] \rightarrow \mathbb{R}$ be a differentiable mapping on $(a, b)$ whose derivative is bounded on $(a, b)$ and denote $\left\|f^{\prime}\right\|_{\infty}=\sup _{t \in(a, b)}\left|f^{\prime}(t)\right|<\infty$. Then for all $x \in[a, b]$ we have

$$
\left|f(x)-\frac{1}{b-a} \int_{a}^{b} f(t) d t\right| \leq\left[\frac{1}{4}+\frac{\left(x-\frac{a+b}{2}\right)^{2}}{(b-a)^{2}}\right](b-a)\left\|f^{\prime}\right\|_{\infty} .
$$

The constant $\frac{1}{4}$ is sharp in the sense that it cannot be replaced by a smaller one.

In [2], Guessab and Schmeisser proved the following companion of Ostrowski's inequality.

Theorem 1.2 Let $f:[a, b] \rightarrow \mathbb{R}$ be satisfying the Lipschitz condition, i.e., $|f(t)-f(s)| \leq$ $M|t-s|$. Then for all $x \in\left[a, \frac{a+b}{2}\right]$ we have

$$
\left|\frac{f(x)+f(a+b-x)}{2}-\frac{1}{b-a} \int_{a}^{b} f(t) d t\right| \leq\left[\frac{1}{8}+2\left(\frac{x-\frac{3 a+b}{4}}{b-a}\right)^{2}\right](b-a) M .
$$

๑) 2013 Liu et al.; licensee Springer. This is an Open Access article distributed under the terms of the Creative Commons Attribution License (http://creativecommons.org/licenses/by/2.0), which permits unrestricted use, distribution, and reproduction in any medium, provided the original work is properly cited. 
The constant $\frac{1}{8}$ is sharp in the sense that it cannot be replaced by a smaller one. In (1.2), the point $x=\frac{3 a+b}{4}$ gives the best estimator.

Motivated by [2], Dragomir [3] proved some companions of Ostrowski's inequality for absolutely continuous functions. Recently, Alomari [4] studied the companion of Ostrowski inequality (1.2) for differentiable bounded mappings. In [5], Liu established some companions of an Ostrowski-type integral inequality for functions whose first derivatives are absolutely continuous and second derivatives belong to $L^{p}(1 \leq p \leq \infty)$ spaces.

Theorem 1.3 Let $f:[a, b] \rightarrow \mathbb{R}$ be such that $f^{\prime}$ is absolutely continuous on $[a, b]$ and $f^{\prime \prime} \in$ $L^{\infty}[a, b]$. Then for all $x \in\left[a, \frac{a+b}{2}\right]$ we have

$$
\begin{aligned}
& \left|\frac{f(x)+f(a+b-x)}{2}-\left(x-\frac{3 a+b}{4}\right) \frac{f^{\prime}(x)-f^{\prime}(a+b-x)}{2}-\frac{1}{b-a} \int_{a}^{b} f(t) d t\right| \\
& \quad \leq\left[\frac{1}{96}+\frac{1}{2} \frac{\left(x-\frac{3 a+b}{4}\right)^{2}}{(b-a)^{2}}\right](b-a)^{2}\left\|f^{\prime \prime}\right\|_{\infty} .
\end{aligned}
$$

The constant $\frac{1}{96}$ is sharp in the sense that it cannot be replaced by a smaller one.

For other related results, the reader may refer to [6-26] and the references therein.

The main aim of this paper is to establish some companions of perturbed Ostrowskitype inequalities for the case when $f^{\prime \prime} \in L^{1}[a, b], f^{\prime \prime \prime} \in L^{2}[a, b]$ and $f^{\prime \prime} \in L^{2}[a, b]$, respectively. For our purpose, we will use the quadratic kernel function with three sections (see (2.1) below) which was defined by Liu in [5]. The special cases of the results we get offer better estimation than the conventional trapezoidal formula and the midpoint formula. These results can apply to composite quadrature rules in numerical integration and probability density functions. The effectiveness of these applications is also illustrated through several specific examples due to better error estimates.

\section{Main results}

To prove our main results, we need the following lemmas.

Lemma 2.1 [5] Let $f:[a, b] \rightarrow \mathbb{R}$ be such that $f^{\prime}$ is absolutely continuous on $[a, b]$. Denote by $K(x, t):[a, b] \rightarrow \mathbb{R}$ the kernel given by

$$
K(x, t)= \begin{cases}\frac{1}{2}(t-a)^{2}, & t \in[a, x], \\ \frac{1}{2}\left(t-\frac{a+b}{2}\right)^{2}, & t \in(x, a+b-x], \\ \frac{1}{2}(t-b)^{2}, & t \in(a+b-x, b],\end{cases}
$$

then the identity

$$
\begin{aligned}
& \frac{1}{b-a} \int_{a}^{b} K(x, t) f^{\prime \prime}(t) d t \\
& \quad=\frac{1}{b-a} \int_{a}^{b} f(t) d t-\frac{f(x)+f(a+b-x)}{2}+\left(x-\frac{3 a+b}{4}\right) \frac{f^{\prime}(x)-f^{\prime}(a+b-x)}{2}
\end{aligned}
$$

holds. 
Li et al. Journal of Inequalities and Applications 2013, 2013:226

Page 3 of 14

http://www.journalofinequalitiesandapplications.com/content/2013/1/226

2.1 The case when $f^{\prime \prime} \in L^{1}[a, b]$ and is bounded

Theorem 2.1 Let $f:[a, b] \rightarrow \mathbb{R}$ be such that $f^{\prime}$ is absolutely continuous on $[a, b]$. If $f^{\prime \prime} \in$ $L^{1}[a, b]$ and $\gamma \leq f^{\prime \prime}(t) \leq \Gamma, \forall t \in[a, b]$, then for all $x \in\left[a, \frac{a+b}{2}\right]$ we have

$$
\begin{aligned}
& \mid \frac{f(x)+f(a+b-x)}{2}-\left(x-\frac{3 a+b}{4}\right) \frac{f^{\prime}(x)-f^{\prime}(a+b-x)}{2} \\
& \quad+\frac{f^{\prime}(b)-f^{\prime}(a)}{b-a}\left[\frac{1}{2}\left(x-\frac{3 a+b}{4}\right)^{2}+\frac{(b-a)^{2}}{96}\right]-\frac{1}{b-a} \int_{a}^{b} f(t) d t \mid \\
& \quad \leq(S-\gamma)\left[\frac{(b-a)^{2}}{48}+\frac{b-a}{4}\left|x-\frac{3 a+b}{4}\right|\right]
\end{aligned}
$$

and

$$
\begin{aligned}
& \mid \frac{f(x)+f(a+b-x)}{2}-\left(x-\frac{3 a+b}{4}\right) \frac{f^{\prime}(x)-f^{\prime}(a+b-x)}{2} \\
& \quad+\frac{f^{\prime}(b)-f^{\prime}(a)}{b-a}\left[\frac{1}{2}\left(x-\frac{3 a+b}{4}\right)^{2}+\frac{(b-a)^{2}}{96}\right]-\frac{1}{b-a} \int_{a}^{b} f(t) d t \mid \\
& \quad \leq(\Gamma-S)\left[\frac{(b-a)^{2}}{48}+\frac{b-a}{4}\left|x-\frac{3 a+b}{4}\right|\right],
\end{aligned}
$$

where $S=\left(f^{\prime}(b)-f^{\prime}(a)\right) /(b-a)$.

Proof From (2.2) and the facts

$$
\frac{1}{b-a} \int_{a}^{b} f^{\prime \prime}(t) d t=\frac{f^{\prime}(b)-f^{\prime}(a)}{b-a}
$$

and

$$
\frac{1}{b-a} \int_{a}^{b} K(x, t) d t=\frac{1}{2}\left(x-\frac{3 a+b}{4}\right)^{2}+\frac{(b-a)^{2}}{96},
$$

it follows that

$$
\begin{aligned}
& \frac{1}{b-a} \int_{a}^{b} K(x, t) f^{\prime \prime}(t) d t-\frac{1}{(b-a)^{2}} \int_{a}^{b} K(x, t) d t \int_{a}^{b} f^{\prime \prime}(t) d t \\
& =\frac{1}{b-a} \int_{a}^{b} f(t) d t-\frac{f(x)+f(a+b-x)}{2}+\left(x-\frac{3 a+b}{4}\right) \frac{f^{\prime}(x)-f^{\prime}(a+b-x)}{2} \\
& \quad-\frac{f^{\prime}(b)-f^{\prime}(a)}{b-a}\left[\frac{1}{2}\left(x-\frac{3 a+b}{4}\right)^{2}+\frac{(b-a)^{2}}{96}\right] .
\end{aligned}
$$

We denote

$$
R_{n}(x)=\frac{1}{b-a} \int_{a}^{b} K(x, t) f^{\prime \prime}(t) d t-\frac{1}{(b-a)^{2}} \int_{a}^{b} K(x, t) d t \int_{a}^{b} f^{\prime \prime}(t) d t .
$$

If $C \in \mathbb{R}$ is an arbitrary constant, then we have

$$
R_{n}(x)=\frac{1}{b-a} \int_{a}^{b}\left(f^{\prime \prime}(t)-C\right)\left[K(x, t)-\frac{1}{b-a} \int_{a}^{b} K(x, s) d s\right] d t .
$$


Furthermore, we have

$$
\left|R_{n}(x)\right| \leq \frac{1}{b-a} \max _{t \in[a, b]}\left|K(x, t)-\frac{1}{b-a} \int_{a}^{b} K(x, s) d s\right| \int_{a}^{b}\left|f^{\prime \prime}(t)-C\right| d t .
$$

To compute

$$
\begin{aligned}
\max _{t \in[a, b]}\left|K(x, t)-\frac{1}{b-a} \int_{a}^{b} K(x, s) d s\right| \\
=\max \left\{\left|\frac{1}{2}(x-a)^{2}-\left[\frac{1}{2}\left(x-\frac{3 a+b}{4}\right)^{2}+\frac{(b-a)^{2}}{96}\right]\right|,\right. \\
\left.\quad\left|\frac{1}{2}\left(\frac{a+b}{2}-x\right)^{2}-\left[\frac{1}{2}\left(x-\frac{3 a+b}{4}\right)^{2}+\frac{(b-a)^{2}}{96}\right]\right|, \frac{1}{2}\left(x-\frac{3 a+b}{4}\right)^{2}+\frac{(b-a)^{2}}{96}\right\} \\
=\max \left\{\frac{b-a}{24}|6 x-5 a-b|, \frac{b-a}{12}|3 x-2 a-b|, \frac{1}{2}\left(x-\frac{3 a+b}{4}\right)^{2}+\frac{(b-a)^{2}}{96}\right\},
\end{aligned}
$$

we denote

$$
\begin{aligned}
& y_{1}=\frac{b-a}{24}|6 x-5 a-b|, \quad y_{2}=\frac{b-a}{12}|3 x-2 a-b|, \\
& y_{3}=\frac{1}{2}\left(x-\frac{3 a+b}{4}\right)^{2}+\frac{(b-a)^{2}}{96} .
\end{aligned}
$$

If we choose $y_{1}=0$, then we get $x_{1}=\frac{5 a+b}{6}$. If we choose $y_{2}=0$, then we get $x_{2}=\frac{2 a+b}{3}$. A direct computation gives that

$$
\begin{cases}y_{2} \geq \max \left\{y_{1}, y_{3}\right\}, & x \in\left[a, \frac{3 a+b}{4}\right], \\ y_{1}>\max \left\{y_{2}, y_{3}\right\}, & x \in\left(\frac{3 a+b}{4}, \frac{a+b}{2}\right] .\end{cases}
$$

Therefore, we get

$$
\begin{aligned}
& \max _{t \in[a, b]}\left|K(x, t)-\frac{1}{b-a} \int_{a}^{b} K(x, s) d s\right| \\
& \quad=\max \left\{y_{1}, y_{2}\right\}=\frac{(b-a)^{2}}{48}+\frac{b-a}{4}\left|x-\frac{3 a+b}{4}\right| .
\end{aligned}
$$

We also have

$$
\int_{a}^{b}\left|f^{\prime \prime}(t)-\gamma\right| d t=(S-\gamma)(b-a)
$$

and

$$
\int_{a}^{b}\left|f^{\prime \prime}(t)-\Gamma\right| d t=(\Gamma-S)(b-a)
$$

Therefore, we obtain (2.3) and (2.4) by using (2.7)-(2.10), (2.1)-(2.15) and choosing $C=\gamma$ and $C=\Gamma$ in (2.10), respectively. 
Corollary 2.1 Under the assumptions of Theorem 2.1, choose

(1) $x=\frac{3 a+b}{4}$, we have

$$
\begin{aligned}
& \left|\frac{f\left(\frac{3 a+b}{4}\right)+f\left(\frac{a+3 b}{4}\right)}{2}+\frac{f^{\prime}(b)-f^{\prime}(a)}{b-a} \frac{(b-a)^{2}}{96}-\frac{1}{b-a} \int_{a}^{b} f(t) d t\right| \\
& \quad \leq \frac{1}{48}(S-\gamma)(b-a)^{2} \\
& \left|\frac{f\left(\frac{3 a+b}{4}\right)+f\left(\frac{a+3 b}{4}\right)}{2}+\frac{f^{\prime}(b)-f^{\prime}(a)}{b-a} \frac{(b-a)^{2}}{96}-\frac{1}{b-a} \int_{a}^{b} f(t) d t\right| \\
& \quad \leq \frac{1}{48}(\Gamma-S)(b-a)^{2} .
\end{aligned}
$$

(2) $x=a$, we have

$$
\begin{aligned}
& \left|\frac{f(a)+f(b)}{2}-\frac{f^{\prime}(b)-f^{\prime}(a)}{b-a} \frac{(b-a)^{2}}{12}-\frac{1}{b-a} \int_{a}^{b} f(t) d t\right| \leq \frac{1}{12}(S-\gamma)(b-a)^{2}, \\
& \left|\frac{f(a)+f(b)}{2}-\frac{f^{\prime}(b)-f^{\prime}(a)}{b-a} \frac{(b-a)^{2}}{12}-\frac{1}{b-a} \int_{a}^{b} f(t) d t\right| \leq \frac{1}{12}(\Gamma-S)(b-a)^{2} .
\end{aligned}
$$

(3) $x=\frac{a+b}{2}$, we have

$$
\begin{aligned}
& \left|f\left(\frac{a+b}{2}\right)+\frac{f^{\prime}(b)-f^{\prime}(a)}{b-a} \frac{(b-a)^{2}}{24}-\frac{1}{b-a} \int_{a}^{b} f(t) d t\right| \leq \frac{1}{12}(S-\gamma)(b-a)^{2}, \\
& \left|f\left(\frac{a+b}{2}\right)+\frac{f^{\prime}(b)-f^{\prime}(a)}{b-a} \frac{(b-a)^{2}}{24}-\frac{1}{b-a} \int_{a}^{b} f(t) d t\right| \leq \frac{1}{12}(\Gamma-S)(b-a)^{3} .
\end{aligned}
$$

Corollary 2.2 Let $f$ be as in Theorem 2.1. Additionally, if $f$ is symmetric about $x=\frac{a+b}{2}$, then for all $x \in\left[a, \frac{a+b}{2}\right]$ we have

$$
\begin{aligned}
\mid f(x) & -\left(x-\frac{3 a+b}{4}\right) f^{\prime}(x)+\frac{f^{\prime}(b)-f^{\prime}(a)}{b-a}\left[\frac{1}{2}\left(x-\frac{3 a+b}{4}\right)^{2}+\frac{(b-a)^{2}}{96}\right] \\
& -\frac{1}{b-a} \int_{a}^{b} f(t) d t \mid \\
\leq & (S-\gamma)\left[\frac{(b-a)^{2}}{48}+\frac{b-a}{4}\left|x-\frac{3 a+b}{4}\right|\right]
\end{aligned}
$$

and

$$
\begin{aligned}
\mid f(x) & -\left(x-\frac{3 a+b}{4}\right) f^{\prime}(x)+\frac{f^{\prime}(b)-f^{\prime}(a)}{b-a}\left[\frac{1}{2}\left(x-\frac{3 a+b}{4}\right)^{2}+\frac{(b-a)^{2}}{96}\right] \\
& -\frac{1}{b-a} \int_{a}^{b} f(t) d t \mid \\
\leq & (\Gamma-S)\left[\frac{(b-a)^{2}}{48}+\frac{b-a}{4}\left|x-\frac{3 a+b}{4}\right|\right] .
\end{aligned}
$$




\subsection{The case when $f^{\prime \prime \prime} \in L^{2}[a, b]$}

Theorem 2.2 Let $f:[a, b] \rightarrow \mathbb{R}$ be a thrice continuously differentiable mapping in $(a, b)$ with $f^{\prime \prime \prime} \in L^{2}[a, b]$. Then for all $x \in\left[a, \frac{a+b}{2}\right]$ we have

$$
\begin{aligned}
& \mid \frac{f(x)+f(a+b-x)}{2}-\left(x-\frac{3 a+b}{4}\right) \frac{f^{\prime}(x)-f^{\prime}(a+b-x)}{2} \\
& \quad+\frac{f^{\prime}(b)-f^{\prime}(a)}{b-a}\left[\frac{1}{2}\left(x-\frac{3 a+b}{4}\right)^{2}+\frac{(b-a)^{2}}{96}\right]-\frac{1}{b-a} \int_{a}^{b} f(t) d t \mid \\
& \leq \frac{1}{\pi}\left\|f^{\prime \prime \prime}\right\|_{2}\left\{\frac{1}{320}(a+b-2 x)^{5}+\frac{1}{10}(x-a)^{5}\right. \\
& \left.\quad-(b-a)\left[\frac{1}{2}\left(x-\frac{3 a+b}{4}\right)^{2}+\frac{(b-a)^{2}}{96}\right]^{2}\right\}^{1 / 2} .
\end{aligned}
$$

Proof Let $R_{n}(x)$ be defined by (2.8). From (2.7), we get

$$
\begin{aligned}
R_{n}(x)= & \frac{1}{b-a} \int_{a}^{b} f(t) d t-\frac{f(x)+f(a+b-x)}{2}+\left(x-\frac{3 a+b}{4}\right) \frac{f^{\prime}(x)-f^{\prime}(a+b-x)}{2} \\
& -\frac{f^{\prime}(b)-f^{\prime}(a)}{b-a}\left[\frac{1}{2}\left(x-\frac{3 a+b}{4}\right)^{2}+\frac{(b-a)^{2}}{96}\right] .
\end{aligned}
$$

If we choose $C=f^{\prime \prime}((a+b) / 2)$ in (2.9) and use the Cauchy inequality, then we get

$$
\begin{aligned}
&\left|R_{n}(x)\right| \\
& \leq \frac{1}{b-a} \int_{a}^{b}\left|f^{\prime \prime}(t)-f^{\prime \prime}\left(\frac{a+b}{2}\right)\right|\left|K(x, t)-\frac{1}{b-a} \int_{a}^{b} K(x, s) d s\right| d t \\
& \leq \frac{1}{b-a}\left[\int_{a}^{b}\left(f^{\prime \prime}(t)-f^{\prime \prime}\left(\frac{a+b}{2}\right)\right)^{2} d t\right]^{1 / 2} \\
& \quad \times\left[\int_{a}^{b}\left(K(x, t)-\frac{1}{b-a} \int_{a}^{b} K(x, s) d s\right)^{2} d t\right]^{1 / 2} .
\end{aligned}
$$

We can use the Diaz-Metcalf inequality (see $[19, \mathrm{p} .83]$ or $[25, \mathrm{p} .424])$ to get

$$
\int_{a}^{b}\left(f^{\prime \prime}(t)-f^{\prime \prime}\left(\frac{a+b}{2}\right)\right)^{2} d t \leq \frac{(b-a)^{2}}{\pi^{2}}\left\|f^{\prime \prime \prime}\right\|_{2}^{2} .
$$

We also have

$$
\begin{aligned}
& \int_{a}^{b}\left(K(x, t)-\frac{1}{b-a} \int_{a}^{b} K(x, s) d s\right)^{2} d t \\
& \quad=\int_{a}^{b} K(x, t)^{2} d t-(b-a)\left[\frac{1}{2}\left(x-\frac{3 a+b}{4}\right)^{2}+\frac{(b-a)^{2}}{96}\right]^{2} \\
& \quad=\frac{1}{320}(a+b-2 x)^{5}+\frac{1}{10}(x-a)^{5}-(b-a)\left[\frac{1}{2}\left(x-\frac{3 a+b}{4}\right)^{2}+\frac{(b-a)^{2}}{96}\right]^{2} .
\end{aligned}
$$

Therefore, using the above relations, we obtain (2.18).

Corollary 2.3 Under the assumptions of Theorem 2.2, choose 
(1) $x=\frac{3 a+b}{4}$, we have

$$
\begin{aligned}
& \left|\frac{f\left(\frac{3 a+b}{4}\right)+f\left(\frac{a+3 b}{4}\right)}{2}+\frac{f^{\prime}(b)-f^{\prime}(a)}{b-a} \frac{(b-a)^{2}}{96}-\frac{1}{b-a} \int_{a}^{b} f(t) d t\right| \\
& \quad \leq \frac{(b-a)^{5 / 2}}{48 \pi \sqrt{5}}\left\|f^{\prime \prime \prime}\right\|_{2} .
\end{aligned}
$$

(2) $x=a$, we have

$$
\left|\frac{f(a)+f(b)}{2}-\frac{f^{\prime}(b)-f^{\prime}(a)}{b-a} \frac{(b-a)^{2}}{12}-\frac{1}{b-a} \int_{a}^{b} f(t) d t\right| \leq \frac{(b-a)^{5 / 2}}{12 \pi \sqrt{5}}\left\|f^{\prime \prime \prime}\right\|_{2} .
$$

(3) $x=\frac{a+b}{2}$, we have

$$
\left|f\left(\frac{a+b}{2}\right)+\frac{f^{\prime}(b)-f^{\prime}(a)}{b-a} \frac{(b-a)^{2}}{24}-\frac{1}{b-a} \int_{a}^{b} f(t) d t\right| \leq \frac{(b-a)^{5 / 2}}{12 \pi \sqrt{5}}\left\|f^{\prime \prime \prime}\right\|_{2} .
$$

Corollary 2.4 Let $f$ be as in Theorem 2.1. Additionally, if $f$ is symmetric about $x=\frac{a+b}{2}$, i.e., $f(a+b-x)=f(x)$, then for all $x \in\left[a, \frac{a+b}{2}\right]$ we have

$$
\begin{aligned}
\mid f(x) & -\left(x-\frac{3 a+b}{4}\right) f^{\prime}(x)+\frac{f^{\prime}(b)-f^{\prime}(a)}{b-a}\left[\frac{1}{2}\left(x-\frac{3 a+b}{4}\right)^{2}+\frac{(b-a)^{2}}{96}\right] \\
& -\frac{1}{b-a} \int_{a}^{b} f(t) d t \mid \\
\leq & \frac{1}{\pi}\left\|f^{\prime \prime \prime}\right\|_{2}\left\{\frac{1}{320}(a+b-2 x)^{5}+\frac{1}{10}(x-a)^{5}\right. \\
& \left.-(b-a)\left[\frac{1}{2}\left(x-\frac{3 a+b}{4}\right)^{2}+\frac{(b-a)^{2}}{96}\right]^{2}\right\}^{1 / 2} .
\end{aligned}
$$

\subsection{The case when $f^{\prime \prime} \in L^{2}[a, b]$}

Theorem 2.3 Let $f:[a, b] \rightarrow \mathbb{R}$ be such that $f^{\prime}$ is absolutely continuous on $[a, b]$ with $f^{\prime \prime} \in$ $L^{2}[a, b]$. Then for all $x \in\left[a, \frac{a+b}{2}\right]$ we have

$$
\begin{aligned}
& \mid \frac{f(x)+f(a+b-x)}{2}-\left(x-\frac{3 a+b}{4}\right) \frac{f^{\prime}(x)-f^{\prime}(a+b-x)}{2} \\
& \quad+\frac{f^{\prime}(b)-f^{\prime}(a)}{b-a}\left[\frac{1}{2}\left(x-\frac{3 a+b}{4}\right)^{2}+\frac{(b-a)^{2}}{96}\right]-\frac{1}{b-a} \int_{a}^{b} f(t) d t \mid \\
& \leq \frac{\sqrt{\sigma\left(f^{\prime \prime}\right)}}{b-a}\left\{\frac{1}{320}(a+b-2 x)^{5}+\frac{1}{10}(x-a)^{5}\right. \\
& \left.\quad-(b-a)\left[\frac{1}{2}\left(x-\frac{3 a+b}{4}\right)^{2}+\frac{(b-a)^{2}}{96}\right]^{2}\right\}^{1 / 2},
\end{aligned}
$$

where $\sigma\left(f^{\prime \prime}\right)$ is defined by

$$
\sigma\left(f^{\prime \prime}\right)=\left\|f^{\prime \prime}\right\|_{2}^{2}-\frac{\left(f^{\prime}(b)-f^{\prime}(a)\right)^{2}}{b-a}=\left\|f^{\prime \prime}\right\|_{2}^{2}-S^{2}(b-a)
$$

and $S$ is defined in Theorem 2.1. 
Proof Let $R_{n}(x)$ be defined by (2.8). If we choose $C=\frac{1}{b-a} \int_{a}^{b} f^{\prime \prime}(s) d s$ in (2.9) and use the Cauchy inequality and (2.21), then we get

$$
\begin{aligned}
&\left|R_{n}(x)\right| \leq \frac{1}{b-a} \int_{a}^{b}\left|f^{\prime \prime}(t)-\frac{1}{b-a} \int_{a}^{b} f^{\prime \prime}(s) d s\right|\left|K(x, t)-\frac{1}{b-a} \int_{a}^{b} K(x, s) d s\right| d t \\
& \leq \frac{1}{b-a}\left[\int_{a}^{b}\left(f^{\prime \prime}(t)-\frac{1}{b-a} \int_{a}^{b} f^{\prime \prime}(s) d s\right)^{2} d t\right]^{1 / 2} \\
& \quad \times\left[\int_{a}^{b}\left(K(x, t)-\frac{1}{b-a} \int_{a}^{b} K(x, s) d s\right)^{2} d t\right]^{1 / 2} \\
& \leq \frac{\sqrt{\sigma\left(f^{\prime \prime}\right)}}{b-a}\left\{\frac{1}{320}(a+b-2 x)^{5}+\frac{1}{10}(x-a)^{5}\right. \\
&\left.\quad-(b-a)\left[\frac{1}{2}\left(x-\frac{3 a+b}{4}\right)^{2}+\frac{(b-a)^{2}}{96}\right]^{2}\right\}^{1 / 2} .
\end{aligned}
$$

Corollary 2.5 Under the assumptions of Theorem 2.3, choose

(1) $x=\frac{3 a+b}{4}$, we have

$$
\begin{aligned}
& \left|\frac{f\left(\frac{3 a+b}{4}\right)+f\left(\frac{a+3 b}{4}\right)}{2}+\frac{f^{\prime}(b)-f^{\prime}(a)}{b-a} \frac{(b-a)^{2}}{96}-\frac{1}{b-a} \int_{a}^{b} f(t) d t\right| \\
& \quad \leq \frac{(b-a)^{3 / 2}}{48 \sqrt{5}} \sqrt{\sigma\left(f^{\prime \prime}\right)} .
\end{aligned}
$$

(2) $x=a$, we have

$$
\left|\frac{f(a)+f(b)}{2}-\frac{f^{\prime}(b)-f^{\prime}(a)}{b-a} \frac{(b-a)^{2}}{12}-\frac{1}{b-a} \int_{a}^{b} f(t) d t\right| \leq \frac{(b-a)^{3 / 2}}{12 \sqrt{5}} \sqrt{\sigma\left(f^{\prime \prime}\right)} .
$$

(3) $x=\frac{a+b}{2}$, we have

$$
\left|f\left(\frac{a+b}{2}\right)+\frac{f^{\prime}(b)-f^{\prime}(a)}{b-a} \frac{(b-a)^{2}}{24}-\frac{1}{b-a} \int_{a}^{b} f(t) d t\right| \leq \frac{(b-a)^{3 / 2}}{12 \sqrt{5}} \sqrt{\sigma\left(f^{\prime \prime}\right)} .
$$

Corollary 2.6 Let $f$ be as in Theorem 2.1. Additionally, if $f$ is symmetric about $x=\frac{a+b}{2}$, then for all $x \in\left[a, \frac{a+b}{2}\right]$ we have

$$
\begin{aligned}
\mid f(x) & -\left(x-\frac{3 a+b}{4}\right) f^{\prime}(x)+\frac{f^{\prime}(b)-f^{\prime}(a)}{b-a}\left[\frac{1}{2}\left(x-\frac{3 a+b}{4}\right)^{2}+\frac{(b-a)^{2}}{96}\right] \\
& -\frac{1}{b-a} \int_{a}^{b} f(t) d t \mid \\
\leq & \frac{\sqrt{\sigma\left(f^{\prime \prime}\right)}}{b-a}\left\{\frac{1}{320}(a+b-2 x)^{5}+\frac{1}{10}(x-a)^{5}\right. \\
& \left.-(b-a)\left[\frac{1}{2}\left(x-\frac{3 a+b}{4}\right)^{2}+\frac{(b-a)^{2}}{96}\right]^{2}\right\}^{1 / 2} .
\end{aligned}
$$




\section{Application to composite quadrature rules}

Let $I_{n}: a=x_{0}<x_{1}<\cdots<x_{n-1}<x_{n}=b$ be a partition of the interval $[a, b]$ and $h_{i}=x_{i+1}-x_{i}$ $(i=0,1,2, \ldots, n-1)$.

Consider the perturbed composite quadrature rules

$$
Q_{n}^{1}\left(I_{n}, f\right)=\frac{1}{2} \sum_{i=0}^{n-1}\left[f\left(\frac{3 x_{i}+x_{i+1}}{4}\right)+f\left(\frac{x_{i}+3 x_{i+1}}{4}\right)\right] h_{i}+\sum_{i=0}^{n-1} \frac{f^{\prime}\left(x_{i+1}\right)-f^{\prime}\left(x_{i}\right)}{96} h_{i}^{2}
$$

and

$$
Q_{n}^{2}\left(I_{n}, f\right)=\frac{1}{2} \sum_{i=0}^{n-1}\left[f\left(\frac{3 x_{i}+x_{i+1}}{4}\right)+f\left(\frac{x_{i}+3 x_{i+1}}{4}\right)\right] h_{i}+\frac{\Gamma+\gamma}{192} \sum_{i=0}^{n-1} h_{i}^{3} .
$$

The following results hold.

Theorem 3.1 Let $f:[a, b] \rightarrow \mathbb{R}$ be such that $f^{\prime}$ is absolutely continuous on $[a, b]$. If $f^{\prime \prime} \in$ $L^{1}[a, b]$ and $\gamma \leq f^{\prime \prime}(t) \leq \Gamma, \forall t \in[a, b]$, then for all $x \in\left[a, \frac{a+b}{2}\right]$ we have

$$
\int_{a}^{b} f(t) d t=Q_{n}^{1}\left(I_{n}, f\right)+R_{n}^{1}\left(I_{n}, f\right)
$$

where $Q_{n}^{1}\left(I_{n}, f\right)$ is defined by formula (3.1), and the remainder $R_{n}^{1}\left(I_{n}, f\right)$ satisfies the estimate

$$
\left|R_{n}^{1}\left(I_{n}, f\right)\right| \leq \frac{1}{48}(S-\gamma) \sum_{i=0}^{n-1} h_{i}^{3}
$$

and

$$
\left|R_{n}^{1}\left(I_{n}, f\right)\right| \leq \frac{1}{48}(\Gamma-S) \sum_{i=0}^{n-1} h_{i}^{3}
$$

Proof Applying inequality (2.1) and (2.1) to the intervals $\left[x_{i}, x_{i+1}\right]$, we get

$$
\begin{aligned}
& \left|\int_{x_{i}}^{x_{i+1}} f(t) d t-\frac{1}{2}\left[f\left(\frac{3 x_{i}+x_{i+1}}{4}\right)+f\left(\frac{x_{i}+3 x_{i+1}}{4}\right)\right] h_{i}-\frac{f^{\prime}\left(x_{i+1}\right)-f^{\prime}\left(x_{i}\right)}{96} h_{i}^{2}\right| \\
& \quad \leq \frac{1}{48}(S-\gamma) h_{i}^{3}
\end{aligned}
$$

and

$$
\begin{aligned}
& \left|\int_{x_{i}}^{x_{i+1}} f(t) d t-\frac{1}{2}\left[f\left(\frac{3 x_{i}+x_{i+1}}{4}\right)+f\left(\frac{x_{i}+3 x_{i+1}}{4}\right)\right] h_{i}-\frac{f^{\prime}\left(x_{i+1}\right)-f^{\prime}\left(x_{i}\right)}{96} h_{i}^{2}\right| \\
& \quad \leq \frac{1}{48}(\Gamma-S) h_{i}^{3}
\end{aligned}
$$

for $i=0,1,2, \ldots, n-1$. Now summing over $i$ from 0 to $n-1$ and using the triangle inequality, we get (3.3) and (3.4). 
Theorem 3.2 Let $f:[a, b] \rightarrow \mathbb{R}$ be a thrice continuously differentiable mapping in $(a, b)$ with $f^{\prime \prime \prime} \in L^{2}[a, b]$. Then for all $x \in\left[a, \frac{a+b}{2}\right]$ we have

$$
\int_{a}^{b} f(t) d t=Q_{n}^{1}\left(I_{n}, f\right)+R_{n}^{1}\left(I_{n}, f\right)
$$

where $Q_{n}^{1}\left(I_{n}, f\right)$ is defined by formula $(3.1)$, and the remainder $R_{n}^{1}\left(I_{n}, f\right)$ satisfies the estimate

$$
\left|R_{n}^{1}\left(I_{n}, f\right)\right| \leq \frac{\left\|f^{\prime \prime \prime}\right\|_{2}}{48 \pi \sqrt{5}} \sum_{i=0}^{n-1} h_{i}^{7 / 2} .
$$

Proof Applying inequality (2.3) to the intervals $\left[x_{i}, x_{i+1}\right]$, we get

$$
\begin{aligned}
& \left|\int_{x_{i}}^{x_{i+1}} f(t) d t-\frac{1}{2}\left[f\left(\frac{3 x_{i}+x_{i+1}}{4}\right)+f\left(\frac{x_{i}+3 x_{i+1}}{4}\right)\right] h_{i}-\frac{f^{\prime}\left(x_{i+1}\right)-f^{\prime}\left(x_{i}\right)}{96} h_{i}^{2}\right| \\
& \quad \leq \frac{h_{i}^{7 / 2}}{48 \pi \sqrt{5}}\left\|f^{\prime \prime \prime}\right\|_{2}
\end{aligned}
$$

for $i=0,1,2, \ldots, n-1$. Now summing over $i$ from 0 to $n-1$ and using the triangle inequality, we get (3.5).

Theorem 3.3 Let $f:[a, b] \rightarrow \mathbb{R}$ be such that $f^{\prime}$ is absolutely continuous on $[a, b]$ with $f^{\prime \prime} \in$ $L^{2}[a, b]$. Then for all $x \in\left[a, \frac{a+b}{2}\right]$ we have

$$
\int_{a}^{b} f(t) d t=Q_{n}^{1}\left(I_{n}, f\right)+R_{n}^{1}\left(I_{n}, f\right)
$$

where $Q_{n}^{1}\left(I_{n}, f\right)$ is defined by formula (3.1), and the remainder $R_{n}^{1}\left(I_{n}, f\right)$ satisfies the estimate

$$
\left|R_{n}^{1}\left(I_{n}, f\right)\right| \leq \frac{\sqrt{\sigma\left(f^{\prime \prime}\right)}}{48 \sqrt{5}} \sum_{i=0}^{n-1} h_{i}^{5 / 2}
$$

Proof Applying inequality (2.5) to the intervals $\left[x_{i}, x_{i+1}\right]$, we get

$$
\begin{aligned}
& \left|\int_{x_{i}}^{x_{i+1}} f(t) d t-\frac{1}{2}\left[f\left(\frac{3 x_{i}+x_{i+1}}{4}\right)+f\left(\frac{x_{i}+3 x_{i+1}}{4}\right)\right] h_{i}-\frac{f^{\prime}\left(x_{i+1}\right)-f^{\prime}\left(x_{i}\right)}{96} h_{i}^{2}\right| \\
& \quad \leq \frac{h_{i}^{5 / 2}}{48 \sqrt{5}} \sqrt{\sigma\left(f^{\prime \prime}\right)}
\end{aligned}
$$

for $i=0,1,2, \ldots, n-1$. Now summing over $i$ from 0 to $n-1$ and using the triangle inequality, we get (3.6).

To illustrate the effectiveness of the perturbed composite quadrature rules (3.1) and (3.2), we compute the approximate values of several specific examples using these two rules and the composite trapezoidal formula

$$
T_{n}\left(I_{n}, f\right)=\frac{h}{2}\left[f(a)+2 \sum_{i=1}^{n-1} f\left(x_{i}\right)+f(b)\right] \text {, }
$$


Table 1 Numerical results

\begin{tabular}{lllrrlll}
\hline $\boldsymbol{f}(\boldsymbol{x})$ & $\boldsymbol{n}$ & {$[\boldsymbol{a}, \boldsymbol{b}]$} & $\boldsymbol{\int}_{\boldsymbol{a}}^{\boldsymbol{b}} \boldsymbol{f}(\mathbf{x}) \boldsymbol{d x}$ & $\boldsymbol{T}_{\boldsymbol{n}}$ & Error of $\boldsymbol{T}_{\boldsymbol{n}}$ & $\boldsymbol{Q}_{\boldsymbol{n}}^{\mathbf{1}}$ and $\boldsymbol{Q}_{\boldsymbol{n}}^{\mathbf{2}}$ & Error of $\boldsymbol{Q}_{\boldsymbol{n}}^{\mathbf{1}}$ and $\boldsymbol{Q}_{\boldsymbol{n}}^{\mathbf{2}}$ \\
\hline $\cos x-x$ & 20 & {$\left[0, \frac{\pi}{2}\right]$} & -0.233701 & -0.234215 & $5.14 \mathrm{E}-4$ & -0.233636 & $6.5 \mathrm{E}-5$ \\
$e^{2 x} \cos \left(e^{x}\right)$ & 20 & {$[0,1]$} & -1.176887 & -1.181466 & $4.579 \mathrm{E}-3$ & -1.176316 & $5.71 \mathrm{E}-4$ \\
$\frac{1}{x^{4}+4 x^{2}+3}$ & 10 & {$[0,1]$} & 0.241549 & 0.241393 & $1.56 \mathrm{E}-4$ & 0.241569 & $2 \mathrm{E}-5$ \\
$\tan x+x$ & 20 & {$\left[0, \frac{\pi}{4}\right]$} & 0.654999 & 0.655127 & $1.28 \mathrm{E}-4$ & 0.654983 & $7 \mathrm{E}-6$ \\
$\ln \left(x^{2}+1\right)$ & 20 & {$[-1,1]$} & 0.527887 & 0.529554 & $1.667 \mathrm{E}-3$ & 0.527679 & $2.08 \mathrm{E}-4$ \\
\hline
\end{tabular}

respectively, and then we compare their errors. We get Table 1, from which the power of these two rules in numerical integration is demonstrated due to better error estimates.

\section{Application to probability density functions}

Now, let $X$ be a random variable taking values in the finite interval $[a, b]$, with the probability density function $f:[a, b] \rightarrow[0,1]$ and with the cumulative distribution function

$$
F(x)=\operatorname{Pr}(X \leq x)=\int_{a}^{x} f(t) d t .
$$

The following results hold.

Theorem 4.1 With the assumptions of Theorem 2.1, we have

$$
\begin{aligned}
& \mid \frac{1}{2}[F(x)+F(a+b-x)]-\left(x-\frac{3 a+b}{4}\right) \frac{f(x)-f(a+b-x)}{2} \\
& \quad+\frac{f(b)-f(a)}{b-a}\left[\frac{1}{2}\left(x-\frac{3 a+b}{4}\right)^{2}+\frac{(b-a)^{2}}{96}\right]-\frac{b-E(X)}{b-a} \mid \\
& \quad \leq(S-\gamma)\left[\frac{(b-a)^{2}}{48}+\frac{b-a}{4}\left|x-\frac{3 a+b}{4}\right|\right]
\end{aligned}
$$

and

$$
\begin{aligned}
& \mid \frac{1}{2}[F(x)+F(a+b-x)]-\left(x-\frac{3 a+b}{4}\right) \frac{f(x)-f(a+b-x)}{2} \\
& \quad+\frac{f(b)-f(a)}{b-a}\left[\frac{1}{2}\left(x-\frac{3 a+b}{4}\right)^{2}+\frac{(b-a)^{2}}{96}\right]-\frac{b-E(X)}{b-a} \mid \\
& \quad \leq(\Gamma-S)\left[\frac{(b-a)^{2}}{48}+\frac{b-a}{4}\left|x-\frac{3 a+b}{4}\right|\right]
\end{aligned}
$$

for all $x \in\left[a, \frac{a+b}{2}\right]$, where $E(X)$ is the expectation of $X$.

Proof By (2.3) and (2.4) on choosing $f=F$ and taking into account

$$
E(X)=\int_{a}^{b} t d F(t)=b-\int_{a}^{b} F(t) d t
$$

we obtain (4.1) and (4.2). 
Corollary 4.1 Under the assumptions of Theorem 4.1 with $x=\frac{3 a+b}{4}$, we have

$$
\begin{aligned}
& \left|\frac{1}{2}\left[F\left(\frac{3 a+b}{4}\right)+F\left(\frac{a+3 b}{4}\right)\right]+\frac{b-a}{96}[f(b)-f(a)]-\frac{b-E(x)}{b-a}\right| \\
& \quad \leq \frac{1}{48}(S-\gamma)(b-a)^{2}
\end{aligned}
$$

and

$$
\begin{aligned}
& \left|\frac{1}{2}\left[F\left(\frac{3 a+b}{4}\right)+F\left(\frac{a+3 b}{4}\right)\right]+\frac{b-a}{96}[f(b)-f(a)]-\frac{b-E(x)}{b-a}\right| \\
& \quad \leq \frac{1}{48}(\Gamma-S)(b-a)^{2} .
\end{aligned}
$$

Theorem 4.2 With the assumptions of Theorem 2.2, we have

$$
\begin{aligned}
& \mid \frac{1}{2}[F(x)+F(a+b-x)]-\left(x-\frac{3 a+b}{4}\right) \frac{f(x)-f(a+b-x)}{2} \\
& \quad+\frac{f(b)-f(a)}{b-a}\left[\frac{1}{2}\left(x-\frac{3 a+b}{4}\right)^{2}+\frac{(b-a)^{2}}{96}\right]-\frac{b-E(X)}{b-a} \mid \\
& \leq \frac{1}{\pi}\left\|f^{\prime \prime \prime}\right\|_{2}\left\{\frac{1}{320}(a+b-2 x)^{5}+\frac{1}{10}(x-a)^{5}\right. \\
& \left.\quad-(b-a)\left[\frac{1}{2}\left(x-\frac{3 a+b}{4}\right)^{2}+\frac{(b-a)^{2}}{96}\right]^{2}\right\}^{1 / 2}
\end{aligned}
$$

for all $x \in\left[a, \frac{a+b}{2}\right]$, where $E(X)$ is the expectation of $X$.

Proof By (2.18) on choosing $f=F$ and taking into account

$$
E(X)=\int_{a}^{b} t d F(t)=b-\int_{a}^{b} F(t) d t
$$

we obtain (4.5).

Corollary 4.2 Under the assumptions of Theorem 4.2 with $x=\frac{3 a+b}{4}$, we have

$$
\begin{aligned}
& \left|\frac{1}{2}\left[F\left(\frac{3 a+b}{4}\right)+F\left(\frac{a+3 b}{4}\right)\right]+\frac{b-a}{96}[f(b)-f(a)]-\frac{b-E(x)}{b-a}\right| \\
& \quad \leq \frac{(b-a)^{5 / 2}}{48 \pi \sqrt{5}}\left\|f^{\prime \prime \prime}\right\|_{2} .
\end{aligned}
$$

Theorem 4.3 With the assumptions of Theorem 2.3, we have

$$
\begin{aligned}
& \mid \frac{1}{2}[F(x)+F(a+b-x)]-\left(x-\frac{3 a+b}{4}\right) \frac{f(x)-f(a+b-x)}{2} \\
& \quad+\frac{f(b)-f(a)}{b-a}\left[\frac{1}{2}\left(x-\frac{3 a+b}{4}\right)^{2}+\frac{(b-a)^{2}}{96}\right]-\frac{b-E(X)}{b-a} \mid \\
& \quad \leq \frac{\sqrt{\sigma\left(f^{\prime \prime}\right)}}{b-a}\left\{\frac{1}{320}(a+b-2 x)^{5}+\frac{1}{10}(x-a)^{5}\right.
\end{aligned}
$$




$$
\left.-(b-a)\left[\frac{1}{2}\left(x-\frac{3 a+b}{4}\right)^{2}+\frac{(b-a)^{2}}{96}\right]^{2}\right\}^{1 / 2}
$$

for all $x \in\left[a, \frac{a+b}{2}\right]$, where $E(X)$ is the expectation of $X$.

Proof By (2.23) on choosing $f=F$ and taking into account

$$
E(X)=\int_{a}^{b} t d F(t)=b-\int_{a}^{b} F(t) d t
$$

we obtain (4.7).

Corollary 4.3 Under the assumptions of Theorem 4.3 with $x=\frac{3 a+b}{4}$, we have

$$
\begin{aligned}
& \left|\frac{1}{2}\left[F\left(\frac{3 a+b}{4}\right)+F\left(\frac{a+3 b}{4}\right)\right]+\frac{b-a}{96}[f(b)-f(a)]-\frac{b-E(x)}{b-a}\right| \\
& \quad \leq \frac{(b-a)^{3 / 2}}{48 \sqrt{5}} \sqrt{\sigma\left(f^{\prime \prime}\right)} .
\end{aligned}
$$

\section{Competing interests}

The authors declare that they have no competing interests.

\section{Authors' contributions}

All authors contributed to the writing of the present article and they read and approved the final manuscript.

\section{Author details}

${ }^{1}$ College of Mathematics and Statistics, Nanjing University of Information Science and Technology, Nanjing, 210044, China. ${ }^{2}$ Department of Mathematics, Hanseo University, Seosan-si, Chungnam-do 356-706, Republic of Korea.

\section{Acknowledgements}

The authors wish to thank the anonymous referees and the editor for their valuable comments. This work was partly supported by the Qing Lan Project of Jiangsu Province, the National Natural Science Foundation of China (Grant No. 41174165) and the Teaching Research Project of NUIST (Grant No. 12JY052).

Received: 1 August 2012 Accepted: 18 April 2013 Published: 6 May 2013

\section{References}

1. Ostrowski, A: Über die Absolutabweichung einer differentiierbaren Funktion von ihrem Integralmittelwert. Comment. Math. Helv. 10(1), 226-227 (1937)

2. Guessab, A, Schmeisser, G: Sharp integral inequalities of the Hermite-Hadamard type. J. Approx. Theory 115(2), 260-288 (2002)

3. Dragomir, SS: Some companions of Ostrowski's inequality for absolutely continuous functions and applications. Bull. Korean Math. Soc. 42(2), 213-230 (2005)

4. Alomari, MW: A companion of Ostrowski's inequality with applications. Transylv. J. Math. Mech. 3(1), 9-14 (2011)

5. Liu, Z: Some companions of an Ostrowski type inequality and applications. JIPAM. J. Inequal. Pure Appl. Math. 10(2), Article ID 52 (2009)

6. Alomari, MW: A companion of Ostrowski's inequality for mappings whose first derivatives are bounded and applications in numerical integration. Transylv. J. Math. Mech. 4(2), 103-109 (2012)

7. Alomari, MW: A generalization of companion inequality of Ostrowski's type for mappings whose first derivatives are bounded and applications in numerical integration. Kragujev. J. Math. 36(1), 77-82 (2012)

8. Barnett, NS, Dragomir, SS, Gomm, I: A companion for the Ostrowski and the generalised trapezoid inequalities. Math. Comput. Model. 50(1-2), 179-187 (2009)

9. Dragomir, SS: A companions of Ostrowski's inequality for functions of bounded variation and applications. RGMIA Res. Rep. Coll. 5, Supp., article 28 (2002)

10. Dragomir, SS: Ostrowski type inequalities for functions defined on linear spaces and applications for semi-inner products. J. Concr. Appl. Math. 3(1), 91-103 (2005)

11. Dragomir, SS: Ostrowski's type inequalities for continuous functions of selfadjoint operators on Hilbert spaces: a survey of recent results. Ann. Funct. Anal. 2(1), 139-205 (2011)

12. Dragomir, SS, Sofo, A: An integral inequality for twice differentiable mappings and applications. Tamkang J. Math. 31(4), 257-266 (2000)

13. Duoandikoetxea, J: A unified approach to several inequalities involving functions and derivatives. Czechoslov. Math J. 51(126)(2), 363-376 (2001) 
14. Huy, VN, Ngô, Q-A: New bounds for the Ostrowski-like type inequalities. Bull. Korean Math. Soc. 48(1), 95-104 (2011)

15. Liu, WJ: Several error inequalities for a quadrature formula with a parameter and applications. Comput. Math. Appl. 56(7), 1766-1772 (2008)

16. Liu, WJ: Some weighted integral inequalities with a parameter and applications. Acta Appl. Math. 109(2), 389-400 (2010)

17. Liu, WJ, Xue, QL, Wang, SF: New generalization of perturbed Ostrowski type inequalities and applications. J. Appl. Math. Comput. 32(1), 157-169 (2010)

18. Liu, Z: Note on a paper by N. Ujević. Appl. Math. Lett. 20(6), 659-663 (2007)

19. Mitrinović, DS, Pečarić, JE, Fink, AM: Inequalities involving functions and their integrals and derivatives. In: Mathematics and Its Applications (East European Series), 53. Kluwer Academic, Dordrecht (1991)

20. Sarikaya, MZ: On the Ostrowski type integral inequality. Acta Math. Univ. Comen. 79(1), 129-134 (2010)

21. Sarikaya, MZ: New weighted Ostrowski and čebyšev type inequalities on time scales. Comput. Math. Appl. 60(5), 1510-1514 (2010)

22. Set, E, Sarıkaya, MZ: On the generalization of Ostrowski and grüss type discrete inequalities. Comput. Math. Appl. 62(1), 455-461 (2011)

23. Tseng, K-L, Hwang, S-R, Dragomir, SS: Generalizations of weighted Ostrowski type inequalities for mappings of bounded variation and their applications. Comput. Math. Appl. 55(8), 1785-1793 (2008)

24. Tseng, K-L, Hwang, S-R, Yang, G-S, Chou, Y-M: Weighted Ostrowski integral inequality for mappings of bounded variation. Taiwan. J. Math. 15(2), 573-585 (2011)

25. Ujević, N: New bounds for the first inequality of Ostrowski-grüss type and applications. Comput. Math. Appl. 46(2-3), 421-427 (2003)

26. Vong, SW: A note on some Ostrowski-like type inequalities. Comput. Math. Appl. 62(1), 532-535 (2011)

doi:10.1186/1029-242X-2013-226

Cite this article as: Liu et al.: Some companions of perturbed Ostrowski-type inequalities based on the quadratic

kernel function with three sections and applications. Journal of Inequalities and Applications 2013 2013:226.

\section{Submit your manuscript to a SpringerOpen ${ }^{\ominus}$ journal and benefit from:}

- Convenient online submission

- Rigorous peer review

- Immediate publication on acceptance

Open access: articles freely available online

High visibility within the field

- Retaining the copyright to your article 